\title{
MARCEL SCHWOB ET LA SIMULATION / DISSIMULATION IDENTITAIRE À TRAVERS LES RECUEILS CEUR DOUBLE ET LE ROI AU MASQUE D'OR
}

\author{
Kévin FRANÇOIS \\ Université de Varsovie
}

\begin{abstract}
En): Simulation and dissimulation are two important writing concepts. Thanks to them, authors can bring their readers into their universe and to their concept of the world. Marcel Schwob in two of his collections of short novels, Coeur double and Le Roi au masque d'or, uses simulation and dissimulation to deepen his own vision of identity and to bring his reader to a similar consciousness: through the depictured stories, he aims to make them feel the presence of others and how close they are.
\end{abstract}

Key words (En): Marcel Schwob ; simulation ; dissimulation ; identity ; otherness ; strange

Mots-clefs (Fr): Marcel Schwob ; simulation ; dissimulation ; identité ; altérité ; étrangeté

\section{Introduction}

Dans son ouvrage L'Acte de lecture. Théorie de l'effet esthétique (1972), Wolfgang ISER déclare qu' "en général, le lecteur du texte de fiction adopte le point de vue du narrateur par "libre mise entre parenthèses de sa faculté critique" » (1976 : 28). Ainsi, par cet abandon partiel de ses facultés critiques, le lecteur entre pleinement dans le monde propre à l'auteur de fiction, il découvre une nouvelle vision du monde à laquelle il s'ouvre lors du processus de lecture jusqu'à même se rendre naif dans une certaine mesure, comme nous le fait remarquer Umberto ECO :

il [le lecteur] choisira une autre attitude interprétative et décidera que le roman lui parle d'un univers un peu étrange où il y a Paris, comme dans le nôtre, mais où la tour Eiffel est faite différemment. Il se préparera éventuellement à accepter l'idée - rien moins - qu'à Paris il n'y a ni métro ni Seine mais un lac et un système de voies surélevées dessinées par Moebius. C'est-àdire qu'il fera des prévisions avec les indications que le texte [l'auteur] lui a données à propos du type de monde auquel il doit s'attendre. (1985:167)

Cette ouverture du lecteur est nécessaire à la mise en place du processus de lecture et permet à l'auteur de créer des effets de lecture. Ces effets sont réalisés à partir d'une vision du lecteur particulière à l'auteur, que l'on peut nommer « lecteur implicite » selon ISER (1976: 75) ou encore «Lecteur Modèle » pour ECO (1985: 80). Ces projections conçues au moment de la rédaction influencent l'auteur dans la construction de son texte, mais «prévoir son Lecteur Modèle ne signifie pas uniquement "espérer" qu'il existe, cela signifie aussi agir sur le texte de façon à le construire » (ECO, 1985: 72), et les effets de lecture sont ainsi présents pour guider et conditionner, jusqu'à un certain point, la personne lisant le texte. Ils impliquent que l'auteur nous conduit dans son monde, que le texte est construit de telle façon qu'il peut aller jusqu'à provoquer des réactions affectives ou cognitives chez le lecteur. 
L'un des outils favorisant ces effets de lecture est la dissimulation que nous comprendrons en l'occurrence comme un phénomène qui se rapporte aux éléments dissimulés du texte. Il s'agit bien ici des «zones d'indétermination» de Roman INGARDEN (1983 : 192) ou des «blancs » de Wolfgang ISER (1976:199), soit des omissions volontaires de l'auteur stimulant les processus de lecture et imaginatif. Cette dissimulation possède un complément : la simulation, qui peut se définir par ce que l'on feint ou ce que l'on incarne, où les notions de duplicité et de mystification se laissent entendre.

Ces phénomènes de dissimulation et de simulation à travers la fiction sont flagrants chez Marcel Schwob qui a su jouer au cours de ses œuvres avec des identités multiples, et bien souvent anonymes, contrairement aux personnages que l'on peut trouver chez les autres conteurs de la même époque comme Bernard Lazare, Henri de Régnier ou Rémy de Gourmont, ce dernier se réfugiant par exemple dans un idéalisme que l'on retrouve aussi chez Schopenhauer, dont la pensée a profondément influencé de nombreux auteurs de la fin du Décadentisme. Schwob, auteur français du XIX ${ }^{\mathrm{e}}$ siècle n'est pas des plus faciles à saisir et s'est souvent vu rattaché au mouvement symboliste, notamment à travers ses nombreux contes. Il est en effet possible de le rapprocher de ce courant et de ses procédés sur certains points comme le fait Léon Blum cité par Hubert Juin :

Il n'est symboliste qu'en raison des procédés de son art. Pour peindre des individus, des passions, des moments de la nature, Schwob n'a jamais recours à l'analyse ou à la description. Son but n'est pas de donner au lecteur la connaissance discursive, la vision détaillée et minutieuse de l'objet choisi. Point du tout ; il s'attache à démêler les saillies excessives, les mouvements représentatifs, les aspects frappants et singuliers qui font de tel être, de tel objet, une réalité particulière et distincte de toutes les autres. Et alors il ne désigne plus les objets ou les êtres que par ce caractère distinctif qui les évoque et les «personnalise » à la fois, qui en est le symbole, c'est-à-dire l'expression équivalente et abrégée. (SCHWOB, 1979 : 25-26)

Il nous faut néanmoins prêter attention à certaines objections qui ont été faites à ce sujet, comme celle de Monique Jutrin pour qui Marcel Schwob est un auteur qui a su dénoncer les limites du symbolisme de manières subtiles : «L'étiquette de "symboliste" qu'on lui accola souvent ne lui convient guère ; à y regarder de plus près, on s'aperçoit que, dans son essence, elle va souvent à l'encontre du symbolisme, même si elle en revêt parfois les apparences » (JUTRIN,1982 : 40). Déclaration illustrée par son analyse du conte « Le Roi au masque d'or », lequel

revêtant les apparences du symbolisme, [...] en démystifie le message. Là où les critiques n'ont voulu voir qu' "imitation", il y a condamnation implicite d'un art qui refuse la vie. Il passa le plus souvent inaperçu, l'anti-symbolisme de Schwob, et l'on passa sous silence sa révolte contre une poétique stérile. (JUTRIN, $1982: 83$ )

De ces deux appréciations ressort l'image d'un Marcel Schwob à plusieurs visages aimant tisser des masques et misant sur la duplicité, si ce n'est sur la multiplicité, pour toujours offrir à son lecteur des messages clairs, mais qui recèlent une complexité et une profondeur qui nécessitent une observation critique. 
Conscient de la multiplicité qui caractérise toute identité (la sienne comprise ${ }^{1}$ ), Schwob apparaît comme un écrivain capable de simuler, dans le sens de «feindre », toute sorte d'identité. Son jeu ne se manifeste pas uniquement dans sa manière, directe ou biaisée, d'adhérer ou non au courant symboliste, mais aussi dans le fait de mettre en place, dans ses œuvres, des formes de simulation de vie qui doivent amener ses lecteurs à l'idée d'une identité multiple. D'ailleurs, les titres des ouvrages dont nous nous sommes servi pour réaliser cette étude pourraient à eux seuls le laisser entendre: Coeur double (1891) et Le Roi au masque d'or (1892) où nous percevons bien le jeu des apparences et l'importance de ce qui ne semble être que suggéré.

\section{La simulation : une voie vers la prise de conscience}

\subsection{Marcel Schwob et la conscience d'identités multiples}

Les identités avec lesquelles l'auteur joue dans ses contes sont une partie intégrante des simulations créées dans un souci de révélation philosophique. Mais n'est-ce pas justement la conscience de cette multiplicité d'identités inhérentes à l'être humain qui pousse Schwob si clairement vers la simulation et la multiplication d'identités à revêtir dans ses écrits? L'auteur de Cour double et du Roi au masque d'or, riche de cette connaissance de lui-même et de ses semblables, la mobilise au sein de ses contes dans un but bien précis pour le lecteur.

Car même si ce sentiment d'étrangeté le trouble, il en joue allègrement et ouvre à le transmettre. À travers ses contes, il nous donne l'impression de cultiver cette multiplicité qu'il peut créer et veut faire sentir, et ce par la simple richesse de son imagination. Faculté qu'il place, comme Baudelaire ou, après lui, des Esseintes de Huysmans, au-dessus de toutes les autres, mais à condition d'être soutenue par la volonté. Ce Marcel Schwob maladif qui rêvait d'aventures (dont témoigne sa fervente admiration pour Stevenson) a trouvé un moyen de se recréer afin de pouvoir les vivre et de les faire vivre. Nous voyons donc bien que Schwob se meut sur le fil d'un dangereux abîme : celui de l'identité et de sa potentielle versatilité qui la rend aussi troublante. Cette potentialité est une richesse qui lui sert à parcourir de nouveaux horizons, qu'il mobilise pour son lecteur. Dans la préface de Cœur double, il définit comme telles ses intentions d'écriture :

Ainsi est atteint le but de ce livre, qui est de mener par le chemin du cœur et par le chemin de

l'histoire de la terreur à la pitié, de montrer que les événements du monde extérieur peuvent être

\footnotetext{
${ }^{1}$ Monique Jutrin signale l'existence d'une lettre de Schwob à Anatole France où il reconnaît se sentir coupable lorsqu'il reçoit des louanges, ayant l'impression de les voler. Nous nous rendons compte à travers ses déclarations que l'auteur considère l'objet de ces éloges comme n'étant pas de lui, mais plutôt d'une partie étrangère à lui-même, une partie sur laquelle il ne parvient pas à exercer un plein contrôle, et que d'ailleurs après 1897 , il semble aussi ne plus pouvoir mobiliser à son gré comme cela transparaît lorsqu'il écrit à sa mère qu'il n'est plus capable de mobiliser ses capacités d'invention. Marcel Schwob semble expérimenter ce que cette formule de Rimbaud résume bien : « Je est un autre », et de là un sentiment d'être étranger à lui-même qui semble l'étreindre.
} 
parallèles aux émotions du monde intérieur, de faire pressentir que dans une seconde de vie intense nous revivons virtuellement et actuellement l'univers. (SCHWOB, 1979 : 38)

Là est le véritable objectif de Schwob: à travers de multiples contes et de multiples identités, mettre en place de véritables simulations permettant à luimême et à ses lecteurs de parcourir l'humanité dans sa diversité et de s'ouvrir ainsi aux autres. L'auteur de Cour double et du Roi au masque d'or conduit ainsi son lecteur dans son univers où ce dernier peut se rendre compte de l'altérité qui le compose pour vivre au mieux avec autrui.

\subsection{La problématique identitaire au sein de l'œuvre}

Pour étayer cette idée selon laquelle Schwob crée dans ses contes des simulations favorisant la conscience d'une identité multiple de tout être humain, penchons-nous d'un peu plus près sur la constitution de ces deux ouvrages. Il est intéressant de constater que dans Le Roi au masque d'or, sur 21 contes, huit sont rédigés à la première personne et parmi ceux-là sept narrateurs sont anonymes ; leur entourage peut être nommé mais eux ne le sont jamais. En plus de ces contes, nous pouvons relever «Le Roi au masque d'or», «Incendie terrestre», «Les Eunuques » et «La Cité dormante » où les personnages ne sont jamais rattachés à un nom, mais toujours à un rôle ou à une fonction (respectivement : roi, Adam et Ève, eunuques et même spadones, Capitaine). Cela est d'autant plus marquant dans Cæeur double où sur 34 contes, 14 sont rédigés à la première personne, et tous ces narrateurs sont anonymes. Cet anonymat se retrouve aussi dans d'autres contes : « Les Sans-gueule », «L'Homme double », «Lilith », « L'Homme gras », «Le conte des oufs », «L'Âge de la pierre polie: La Vendeuse d'Ambre », «L'Epoque romaine: La Moisson Sabine», «Instantanées», «La Terreur future », soit 23 contes au total où le sujet principal de la narration, ou le narrateur, ne possède pas de nom; 35 sur 55, si l'on combine les deux recueils. Pour commenter ce phénomène, Monique JUTRIN emploie la formule de «tragédie du nom» (1982: 75), mais rapporté à l'auteur, ce fait corroborerait les remarques précédentes que nous avons pu énoncer quant à la prise de conscience par Marcel Schwob d'une fragmentation identitaire inhérente à l'être humain. Charles Baudouin, qui s'est intéressé au processus de création artistique, suppose d'ailleurs dans l'œuvre d'un auteur une réponse apportée à un traumatisme vécu (TRIPET, 2002: 78) : la réalisation de cette multiplicité de l'identité chez Schwob. Cette problématique identitaire se laisse ressentir à l'intérieur des contes, non pas que les personnages se plaignent d'une absence de patronyme ou de surnom, mais plusieurs histoires partagent pour thématique une recherche d'identité («Le Roi au masque d'or», «Les Faulx-Visaiges», «L'Homme double», «L'Homme voilé », «Les Noces d'Arz », ou encore «Fleur de cinq-pierres »). Chacun des protagonistes, à sa manière, cherche à être complet ou à définir une identité, mais cela ne s'arrête pas là et cette identité peut aussi se rapporter à la pure apparence comme c'est le cas dans «L'Homme gras » ou «Le Loup », où à la fin de chacun de ces récits le rideau tombe pour laisser apparaître la réelle apparence, soit la nouvelle identité. Cette quête identitaire des personnages est donc le reflet d'une 
problématique récurrente chez Schwob et qu'il veut transmettre à ses lecteurs. En jouant de telle manière sur les identités, il révèle les difficultés à la saisir, mais surtout, il met en place un phénomène de «contamination » (VAX, 1965: 124) favorisant l'identification du lecteur aux personnages mis en scène. Il peut ainsi confronter ceux qui le lisent à l'étrangeté qui est en eux, prise de conscience qui est selon Julia KRISTEVA (1988 : 250) un levier nous permettant de nous accepter et de vivre avec autrui. C'est justement ce que sous-tend la théorie de «la terreur et de la pitié » déjà évoquée, selon laquelle c'est en ayant expérimenté la terreur de notre vacuité que nous pouvons éprouver assez de pitié pour nous ouvrir aux autres et les accepter. Autrement dit, Schwob, conscient de la fragilité de notre identité, emploie le «je» en tant que «terme voyageur», comme le décrit RICEEUR (1990: 65), afin de faire traverser à ses lecteurs toute une gamme de sentiments, toute une myriade de vies, qui le rapprocheraient, à travers ces expériences, de l'autre.

Comme nous avons pu l'établir précédemment, les écrits de Marcel Schwob sont en grande partie guidés par la prise de conscience de son étrangeté à soimême et sa volonté de la partager. Or, Julia KRISTEVA distingue deux types d'étrangers :

D'une part, ceux qui se consument dans l'écartèlement entre ce qui n'est plus et ce qui ne sera jamais: les adeptes du neutre, les partisans du vide; durcis ou larmoyants, mais toujours désillusionnés; pas forcément défaitistes, ils donnent souvent les meilleurs des ironistes. D'autre part, ceux qui transcendent : ni avant ni maintenant, mais au-delà, ils sont tendus dans une passion certes à jamais inassouvie, mais tenace, vers une autre terre toujours promise, celle d'un métier, d'un amour, d'un enfant, d'une gloire. (1988:21)

Assurément, nous pouvons rattacher Schwob au deuxième type et ce grâce à sa volonté, à travers l'écriture, de mettre à profit cette multiplicité identitaire pour vivre et faire vivre de multiples aventures, permettre à ses lecteurs de s'ouvrir aux autres.

\section{La dissimulation pour renforcer l'identification}

\subsection{L'espace figuré et le flou cultivé}

La simulation n'est pas le seul moyen de favoriser des effets de lecture et l'auteur peut aussi avoir recours à la dissimulation. Cette dissimulation permet à son tour une forme de «contamination» à travers l'omission volontaire d'éléments du texte, procédé qui selon Rachel BOUVET permet au lecteur de se réaliser dans le cadre d'une lecture-en-progression :

il ne faut pas perdre de vue le fait que la lecture n'est pas seulement une construction intellectuelle visant l'élaboration d'une signification. Le plaisir ou la déception que l'on ressent au cours de la traversée du texte, le rappel de certains souvenirs, de certaines émotions, font de la lecture un acte intime, un lieu où se construit un véritable complexe émotionnel. (2007: 49) 
Pour faciliter l'identification avec les personnages mis en scène, Marcel Schwob cultive un flou spatio-temporel, comme nous pouvons l'observer avec la plupart des contes des deux recueils. "La Légende des gueux » mise à part, ils sont presque tous situés dans un «jadis » ou un «autrefois », et même si parfois des lieux sont nommés - comme la Libye ou Milet - ils sont présentés comme des lieux d'enchantement et de sortilèges, en dehors de notre temps. Ainsi, en plus de placer son lecteur dans une temporalité volontairement floue, Marcel Schwob jongle de manière admirable avec ce que Roman INGARDEN a nommé les espaces réels, figurés, et représentés (1983: 191). Rappelons que l'espace figuré est celui qui est mis en place et décrit par l'auteur alors que l'espace représenté est celui que fait surgir le lecteur au moment de sa lecture. Ingarden relève à travers ces trois cas que l'essence de l'espace en général est d'être sans discontinuités. Cette déclaration est évidente en ce qui concerne l'espace réel, mais c'est grâce à ce que l'auteur nomme des «zones d'indétermination » que la discontinuité est possible dans les espaces figuré et représenté. Ces zones d'indétermination, couplées à ce que Husserl nomme «le point zéro de l'orientation» (INGARDEN, 1983: 197), Schwob les mobilise afin de guider son lecteur dans un fantastique «tout intérieur » selon les termes d'Anatole France (SCHWOB, 1979: 26), sans spectres ni fantômes, et renforcer par là même le processus de simulation ou d'identification au personnage. C'est en mettant en scène des protagonistes à l'identité peu ou pas définie, dans un lieu et un temps tout aussi flous, et de là propices à l'arbitraire et à l'interprétation, que l'auteur facilite la variation identitaire du lecteur et donc la prise de conscience de sa propre altérité.

\subsection{Exemples de dissimulation dans deux contes : «Le Roi au masque d'or » et « Les Embaumeuses »}

Pour démontrer cela, prenons l'exemple de deux contes du recueil «Le Roi au Masque d'or » : le récit qui a donné son titre à l'ouvrage, et «Les Embaumeuses ». Dans la première de ces histoires, le point zéro d'orientation est constitué par la personne du roi : c'est seulement à travers ses actions et son regard que les espaces figurés nous sont dévoilés (VIBERT, 2002 : 306-307), et ce, même après son énucléation suite à laquelle nous sommes guidés par ces sensations (VIBERT, 2002 : 314). Par ce procédé, le lecteur suit le parcours initiatique du roi qui le conduit à découvrir ce qui se cache sous son masque, sa véritable identité attachée à la maladie, et sous les beaux atours du monde qui l'entoure. De plus, Schwob laisse une grande liberté d'expression à l'espace représenté, multipliant les zones d'indétermination, ne décrivant les lieux dans lesquels le roi se meut que par des remarques colorées et quelques indications esthétiques (VIBERT, 2002:308), pour guider sans s'imposer, ce qui permet à chacun de se glisser derrière ce masque d'or. Une ambiance est créée, pleine de faste et de faux-semblants, une direction est donnée, menant justement le roi et le lecteur à dépasser les apparences.

Cela est renforcé dans le conte intitulé «Les Embaumeuses » où le point zéro d'orientation se trouve être un narrateur interne dont l'identité n'est jamais déclinée, nous connaissons seulement le nom de son frère: Ophélion. Dans ce 
récit, une première zone d'indétermination est mise en place à l'aide d'une description générale du lieu où se déroule l'histoire faite par le narrateur et ses préjugés mystiques : "Qu'il y ait en Libye, sur les confins de l'Ethiopie où vivent les hommes très vieux et très sages, des sorcelleries plus mystérieuses que celles des magiciennes de Thessalie, je ne puis en douter. » (VIBERT, 2002: 334). C'est donc un point de vue arbitraire qui nous est offert, arbitraire et omnipotent car c'est à travers lui et ses croyances que nous découvrons le monde figuré. De plus, les verbes soulignant l'incertitude de ce que le narrateur voit se multiplient comme : «paraître », «apercevoir », « sembler », et cela couplé à des descriptions de lieux où le champ lexical relevant de l'obscurité dispose d'une large part (VIBERT, 2002 : 336-338). C'est donc une découverte de la Libye floue et dirigée que nous propose Marcel Schwob, ce qui laisse, comme dans «Le Roi au Masque d'or », une grande liberté à l'espace représenté du lecteur pour combler les zones d'indétermination et facilite le jeu identitaire où le narrateur de ce récit peut être chacun de nous. Nous nous rendons donc bien compte combien ces jeux sur les espaces que le narrateur parcourt permettent la formation de simulations en laissant le soin au lecteur de meubler ces espaces décrits, sans qu'il ne puisse jamais réellement se les approprier. Des sentiments de familiarité et de liberté sont créés, permettant à chacun une forme d'identification, tout en laissant un sentiment d'étrangeté trôner. Ce type de procédé est d'ailleurs aussi relevé par Wolfgang ISER qui, en partie à l'aide de ses études sur Ingarden, développa ce qu'il nomme les «blancs » du texte :

discontinuité du récit [qui] fait que le lecteur est amené à produire lui-même ce qui rend vivante l'action décrite : il se met à vivre avec les personnages et subit avec eux les événements dans lesquels ils sont impliqués. En effet, son manque d'information sur la suite des événements fait qu'il partage l'incertitude des personnages quant à leur destinée, et cet horizon vide commun aux personnages et aux lecteurs, lie le lecteur au sort des personnages. (1976:333)

Nous nous rendons bien compte qu'à travers ses contes Schwob favorise l'identification possible entre le narrateur et l'auteur ou le lecteur, il met ainsi en place de véritables simulations au service de sa philosophie et de son questionnement identitaire.

\section{Conclusion}

Ainsi, à travers ces deux recueils, Marcel Schwob nous fait voyager au sein de cette étrangeté qu'il ressent et qu'il porte en lui pour nous mener, selon ses termes, de la «terreur à la pitié » et donc vers autrui. Après avoir observé que des effets de lecture peuvent être favorisés par des phénomènes de simulation et de dissimulation, il est devenu apparent que l'auteur de Coeur double et du Roi au masque d'or les mobilise pour faciliter l'identification de ses lecteurs aux

\footnotetext{
${ }^{2}$ Nous avons volontairement omis le recueil Vies imaginaires (1896) de Marcel Schwob où même si une problématique similaire peut être développée, l'angle d'approche et les procédés sont différents. Schwob présentant lui-même dans la préface de son œuvre ce travail comme un exercice de biographie.
} 
personnages mis en scène. Ce recours à une «contamination » sert la philosophie littéraire de Schwob qui est d'ouvrir son lecteur à l'altérité et à la conscience de la diversité de l'humanité. Ainsi, à travers d'habiles procédés, Schwob simule et dissimule pour mieux révéler en impliquant son lecteur, en lui donnant «l'impression de mener une autre vie » (ISER, 1976:231), et il est remarquable que cela se fasse aussi bien dans des contes reconnus comme fantastiques que dans des histoires qui se veulent plus historiques comme celles tirées de «La Légende des gueux ».

\section{BIBLIOGRAPHIE}

BOUVET Rachel (2007), Etranges récits, étranges lectures - Essai sur l'effet fantastique, Québec, Presses de l'Université du Québec.

ECO Umberto (1985), Lector in Fabula ou la coopération interprétative dans les textes narratifs, Paris, Editions Grasset \& Fasquelle.

INGARDEN Roman (1983), L'œuvre d'art littéraire, Lausanne, L'âge d'homme.

ISER Wolfgang (1976), L'Acte de lecture. Théorie de l'effet esthétique, Bruxelles, Pierre Mardaga, éditeur.

JUTRIN Monique (1982), Marcel Schwob : «Cour double », Vevey, Editions de l'Aire.

KRISTEVA Julia (1988), Etrangers à nous-mêmes, Paris, Librairie Arthème Fayard. RICEUR Paul (1990), Soi-même comme un autre, Paris, Editions du Seuil.

Schwob Marcel (1979), Cœur double/ Mimes, Paris, Union Générale d'Éditions.

TRIPET Arnaud (2002), «Les chemins du mythe dans la critique du XX $\mathrm{X}^{\mathrm{e}}$ siècle », in : Perspectives historiques et métacritiques sur la critique littéraire du XX siècle, CHUDAK Henryk (dir.), Varsovie, Publications de L'Institut de Philologie Romane de L'Université de Varsovie.

VAX Louis (1965), La séduction de l'étrange, Paris, Presses Universitaires de France.

VIBERT Bertrand (2009), Contes symbolistes, vol.1, Grenoble, Ellug. 